\title{
A SMALL SCALE CELL CULTURE SYSTEM TO ANALYZE MECHANOBIOLOGY USING REPORTER GENE CONSTRUCTS AND POLYURETHANE DISHES
}

Lothar Seefried $^{1}$, Sigrid Mueller-Deubert ${ }^{1}$, Thomas Schwarz ${ }^{1}$, Thomas Lind ${ }^{2}$, Birgit Mentrup ${ }^{1}$, Melanie Kober ${ }^{1}$, Denitsa Docheva ${ }^{3}$, Astrid Liedert ${ }^{4}$, Moustapha Kassem ${ }^{5}$, Anita Ignatius ${ }^{4}$, Matthias Schieker ${ }^{3}$, Lutz Claes ${ }^{4}$, Winfried Wilke ${ }^{2}$, Franz Jakob ${ }^{1}$, and Regina Ebert ${ }^{1}$

\author{
${ }^{1}$ Orthopedic Center for Musculoskeletal Research, Orthopedic Department, University of Würzburg, Würzburg, \\ Germany \\ ${ }^{2}$ Fachhochschule Würzburg-Schweinfurt, Fakultät Maschinenbau, Schweinfurt, Germany \\ ${ }^{3}$ Experimental Surgery and Regenerative Medicine, Department of Surgery, Ludwig-Maximilians-University (LMU), \\ München, Germany. \\ ${ }^{4}$ Institute of Orthopedic Research and Biomechanics, University of Ulm, Ulm, Germany \\ ${ }^{5}$ Department of Endocrinology, University Hospital of Odense, Odense, Denmark
}

\section{Abstract}

Mechanical forces are translated into biochemical signals and contribute to cell differentiation and phenotype maintenance. Mesenchymal stem cells and their tissuespecific offspring, as osteoblasts and chondrocytes, cells of cardiovascular tissues and lung cells are sensitive to mechanical loading but molecules and mechanisms involved have to be unraveled. It is well established that cellular mechanotransduction is mediated e.g. by activation of the transcription factor SP1 and by kinase signaling cascades resulting in the activation of the AP1 complex. To investigate cellular mechanisms involved in mechanotransduction and to analyze substances, which modulate cellular mechanosensitivity reporter gene constructs, which can be transfected into cells of interest might be helpful. Suitable small-scale bioreactor systems and mechanosensitive reporter gene constructs are lacking. To analyze the molecular mechanisms of mechanotransduction and its crosstalk with biochemically induced signal transduction, AP1 and SP1 luciferase reporter gene constructs were cloned and transfected into various cell lines and primary cells. A newly developed bioreactor and small-scale 24-well polyurethane dishes were used to apply cyclic stretching to the transfected cells. $1 \mathrm{~Hz}$ cyclic stretching for $30 \mathrm{~min}$ in this system resulted in a significant stimulation of AP1 and SP1 mediated luciferase activity compared to unstimulated cells. In summary we describe a small-scale cell culture/bioreactor system capable of analyzing subcellular crosstalk mechanisms in mechanotransduction, mechanosensitivity of primary cells and of screening the activity of putative mechanosensitizers as new targets, e.g. for the treatment of bone loss caused by both disuse and signal transduction related alterations of mechanotransduction.

Keywords: Mechanical strain, mechanosensitive reporter gene constructs, bioreactor.

*Address for correspondence:

Franz Jakob

Orthopedic Center for Musculoskeletal Research

University of Wuerzburg, Brettreichstrasse 11

D-97074 Würzburg, Germany.

Telephone Number: 00499318031580

FAX Number: 00499318031599

E-mail: f-jakob.klh@uni-wuerzburg.de
Mechanical forces stimulate cell differentiation and control/maintain tissue function via membrane associated "mechanoreceptor" mechanisms, activation of second messengers and downstream gene regulation in various mammalian systems like musculoskeletal and cardiovascular tissues (Hahn and Schwartz, 2009; Jani and Schock, 2009; Papachroni et al., 2009). Cell adhesion molecules like integrins, membrane associated receptors and strain sensitive channels have been described to transmit mechanical forces to intracellular structures like the actin cytoskeleton and second messengers like calcium flux (Asparuhova et al., 2009; Huveneers and Danen, 2009; Kiselyov and Patterson, 2009; Puklin-Faucher and Sheetz, 2009; Sharif-Naeini et al., 2010). Mechanobiochemical transformation in single cells is elicited by the stimulation of ERK1/2 kinase phosphorylation cascades, activation and nuclear translocation of transcription factors and modulation of gene expression (Khatiwala et al., 2009; Klossner et al., 2009; Liu et al., 2009; Rangaswami et al., 2009; Young et al., 2009).

As an immediate early effect c-fos is rapidly induced in many systems and probably contributes to steadily increasing signal intensity via activation of the c-fos/jun alias AP1 transcription factor complex. Promoters comprising AP1 response elements have been shown to respond to mechanical strain, as were elements comprising binding sites for SP1 or NFkB (Liedert et al., 2009). A consensus shear stress responsive element SSRE (GAGACC) has been described to be activated especially in systems related to the vasculature (Inoue et al., 2004; Kletsas et al., 2002; Liedert et al., 2006b; Liedert et al., 2009; Miyagi et al., 2005; Ogasawara et al., 2001; Peverali et al., 2001; Silberman et al., 2009; Sumpio et al., 1997).

Mechanotransduction contributes to physiology in that it mediates tissue modeling according to environmental and life style requirements and this applies especially to mesenchymal tissues. Deficient or enhanced mechanotransduction/mechanosensitivity may cause pathology. Osteoporosis is a disease, which is usually caused by a mixture of pathogenetic factors like disuse of the musculoskeletal system, altered bone quality and serious alteration of mechanotransduction processes, which stimulate bone modeling and remodeling according to mechanical loading. A series of genetic as well as agerelated factors may translate this susceptibility into 
a stretching device

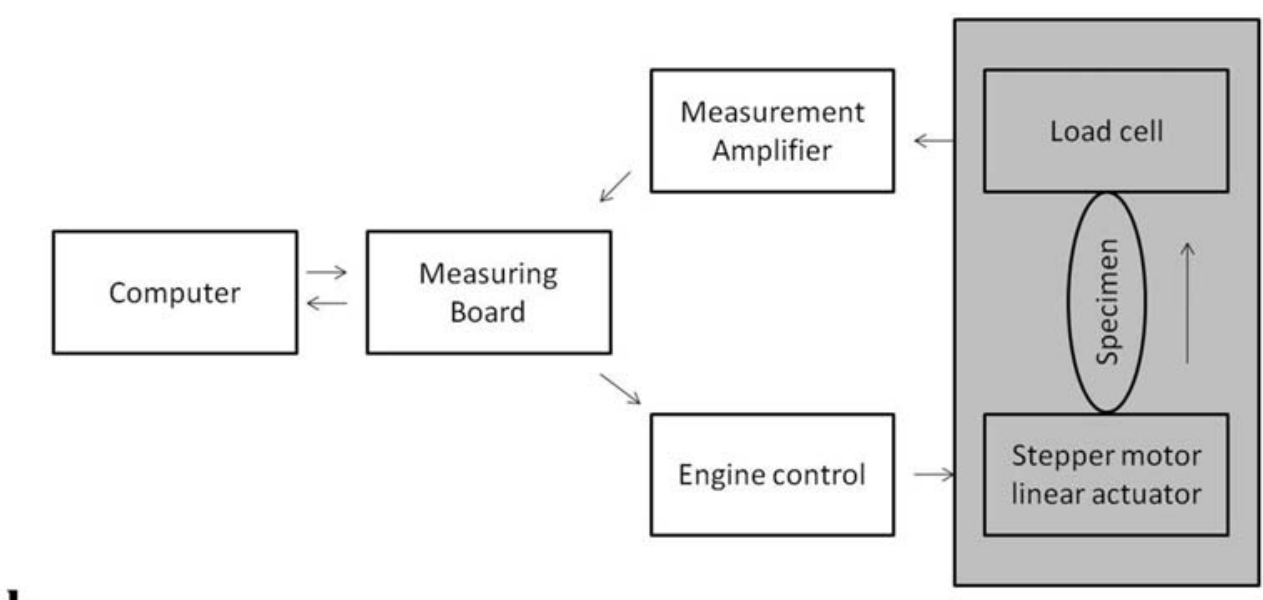

b

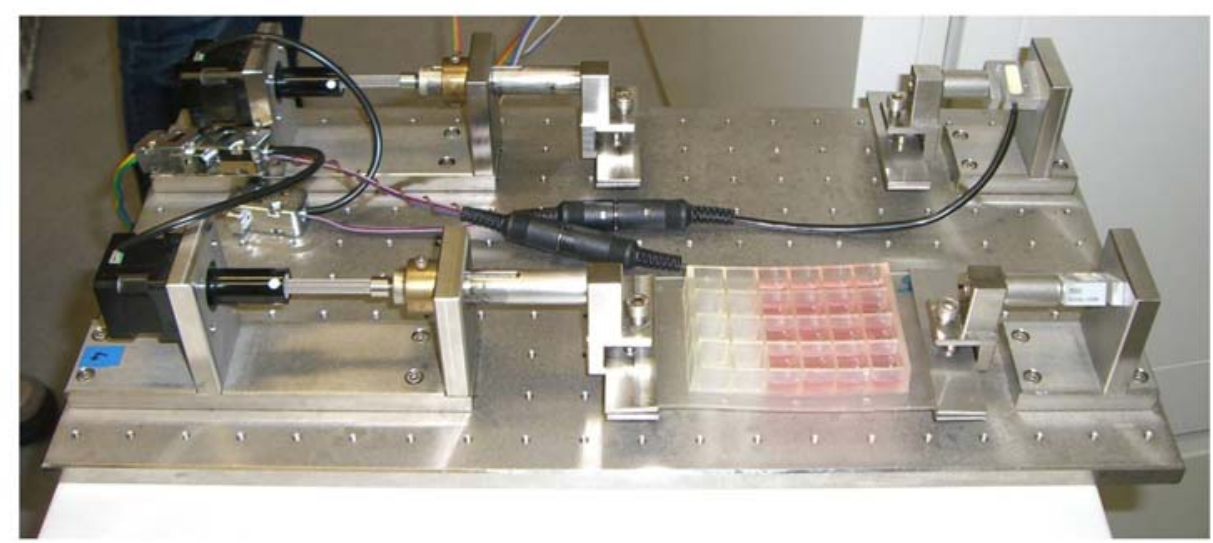

Fig. 1. (a) Schematic illustration of all bioreactor components. (b) Photograph of the newly developed bioreactor. The picture represents the grey shaded area of Fig. 1a.

inadequate bone loss and fragility fractures. The molecular mechanisms, which translate mechanical forces into biochemical signals and their alterations in disease are only partially known (Judex et al., 2009). There are solid data to assume that loss of sex hormone production is a major contributor especially in postmenopausal osteoporosis. Estrogens according to epidemiological data are bone mechanosensitizers, since girls in puberty start to enhance bone mass albeit they do not increase muscle mass accordingly. The molecular basis of this interaction seems to be the interaction of estrogen signaling and the wnt/frz pathway (Armstrong et al., 2007; Liedert et al., 2010).

To further unravel the molecular mechanisms of mechanotransduction in health and disease, the subcellular level of alterations and the respective genomic and proteomic changes, adequate test systems are needed allowing for robust, sensitive, reproducible analyses, which can be performed in a higher throughput in comparison to presently available systems. Moreover experiments using primary cells require material sparing setups. We present here miniaturized 24-well polyurethane dishes with homogenous characteristics for cyclic strain application and a newly designed bioreactor system suitable for applying shear stress to cell cultures. As AP1 and SP1 response elements are mediating mechanical strain in signal transduction cascades these elements were chosen and cloned into luciferase reporter vectors, which can be transfected or lentivirally transduced into various cell lines and primary cells from patients.

\section{Materials and Methods}

\section{Bioreactor design and production}

A multifunctional bioreactor was constructed allowing for flexible resistance adopted cyclic stretching protocols of tissue samples/tissue engineering constructs in media flasks and flexible cell culture dishes (Fig. 1a). A variable fixation device allowed for appropriate anchorage of different specimen within the bioreactor (Fig. 1b). To ensure a high variability in applications, the whole system is build up modularly in a way that the different components of the reactor e.g. the driving module and the force measurement module can be positioned variably on a perforated board with a drilling hole distance of $25 \mathrm{~mm}$. The propulsion module is equipped with an electric linear stepper motor (Series 35000, size 14) and the respective engine control (DCM 8028, both Haydon, Waterbury, CT, USA). The actuator can provide a maximum traction force of $100 \mathrm{~N}$ at a maximum speed of $12.2 \mathrm{~mm} / \mathrm{sec}$. The force measurement module consists of a load cell (KD 24S$100 \mathrm{~N})$ and a measurement amplifier (GSV $11 \mathrm{H}$, both ME Messsysteme, Henningsdorf, Germany), which can detect forces up to $100 \mathrm{~N}$ with a resolution of $\pm 0.1 \mathrm{~N}$. Force sensor and propulsion module / actuator are placed opposite to one another on the perforated plate. The reactor is connected to a computer, which drives the propulsion module and records the forces sensed by the force measurement module during the stretching process. Potential amplitude of the stepper motor ranges from 0 to 
a

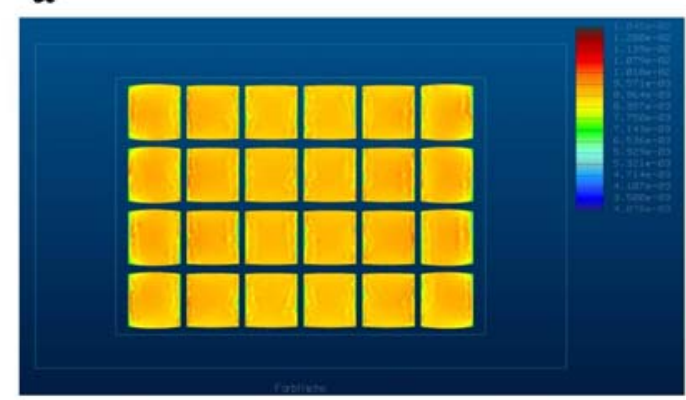

c

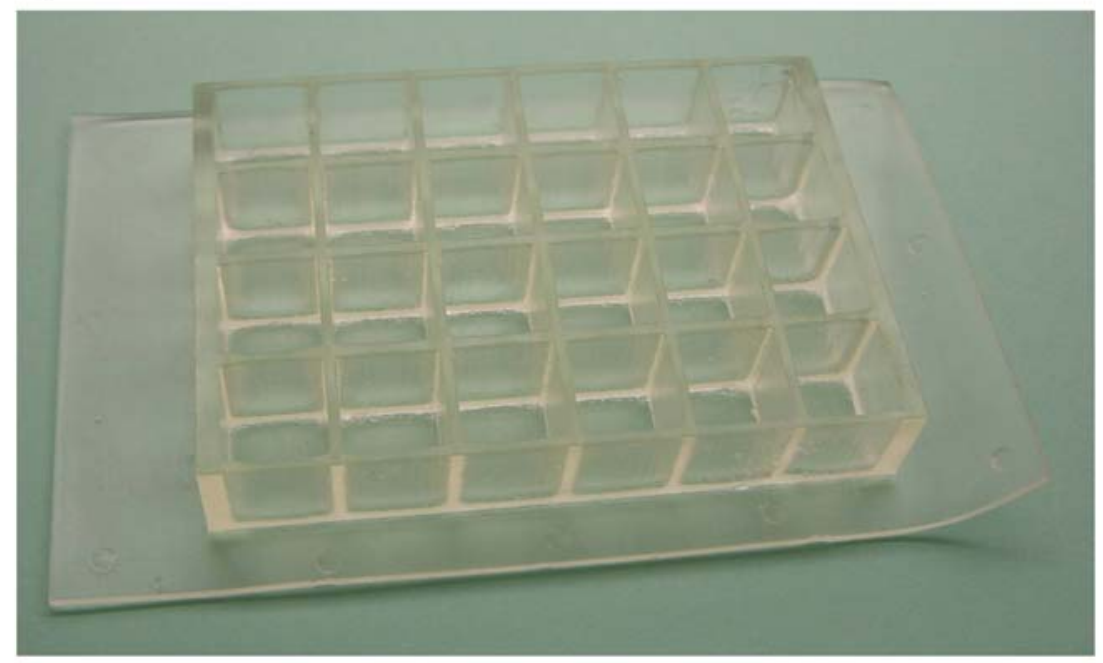

b

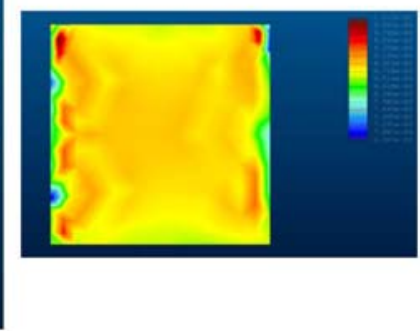

Fig. 2. Finite element analysis of (a) a complete 24-well PU dish and (b) one single well. Identical colors represent equal extension of the $\mathrm{PU}$ material after $1 \%$ cyclic strain application. Red color represents high extension while blue color represents low extension; (c) Photograph of one newly developed polyurethane dish.
$38 \mathrm{~mm}$. Velocity of the excursion can be modified continuously up to $12 \mathrm{~mm} / \mathrm{s}$. Considering these technical specifications, the reactor in combination with the flexible PU dishes described below theoretically enables mechanical stimulation with a maximum strain of about $25 \%(250,000 \mu \varepsilon)$ at $0.5 \mathrm{~Hz}$ and a maximum frequency of about $6 \mathrm{~Hz}$ with $0.5 \%(5000 \mu \varepsilon)$ strain. The modular configuration of the reactor together with the variable fixation device enables mechanical stimulation of specimen with a length from 15 to $150 \mathrm{~mm}$ and a width of up to 85 $\mathrm{mm}$. For secure fixation of the flexible PU culture dishes, a chucking device with coplanar, roughened clamping plates, covering the whole breadth of the dishes was used (Fig. 1b). The bioreactor is designed to operate in a conventional incubator under cell culture conditions $\left(37^{\circ} \mathrm{C}\right.$, $5 \% \mathrm{CO}_{2}$, humidity $95 \%$ ). All metal parts can be sterilized by spray disinfection.

\section{Measurement and control program}

The process of mechanical stimulation and data acquisition is controlled by an individually programmed graphical user interface based on the commercial software LabView (National Instruments Germany, München, Germany). Signals to the actuator and from the measurement module are integrated on a measuring board (cDAQ 9172, National Instruments Germany), which is physically connected to the computer with a conventional USB-cable. Thereby, the complete process of stimulation as well as data acquisition is accomplished by the software. The user interface based on the software allows for an independent controlling of multiple stretching devices. This setting enables application of preassigned stretching protocols with chancing parameters over time, e.g. variation of the oscillating stretching parameters such as amplitude and frequency and the setting of braking rates and pausing at the turning points of oscillation without human intervention. Implementation of a feed-back loop, which continuously integrates the information detected by the force sensor allows for technical surveillance of the actual forces exerted on the specimen, thereby permitting calibration of the system by definition of a reproducible construct preload as starting point for the experiments. Continuous automatic monitoring of the actual values additionally allows - if necessary - adaption of stimulation parameters by the computer in order to ensure constant application of the predefined values for stimulation. Based on data obtained from the sensor, actual forces and current elongation are continuously displayed by two graphs, permitting monitoring of process flow by the user. All data monitored are recorded and can be comprehensively analyzed after the experiment.

\section{Design and fabrication of cell culture dishes}

Design of the multi-well cell culture dishes for mechanical stimulation was aimed at equal strain distribution, both within each single well and between the different chambers across the whole culture dish (Fig. 2a and b). Therefore, construction including FEM calculations was performed with the software ProEngineer Wildfire 2.0 (Parametric Technology Corporation, PTC, Needham, MA, USA). Tetrahedrons and triangles were used as finite elements. Stimulation parameters used for FEM calculation were 1 
$\mathbf{a}$

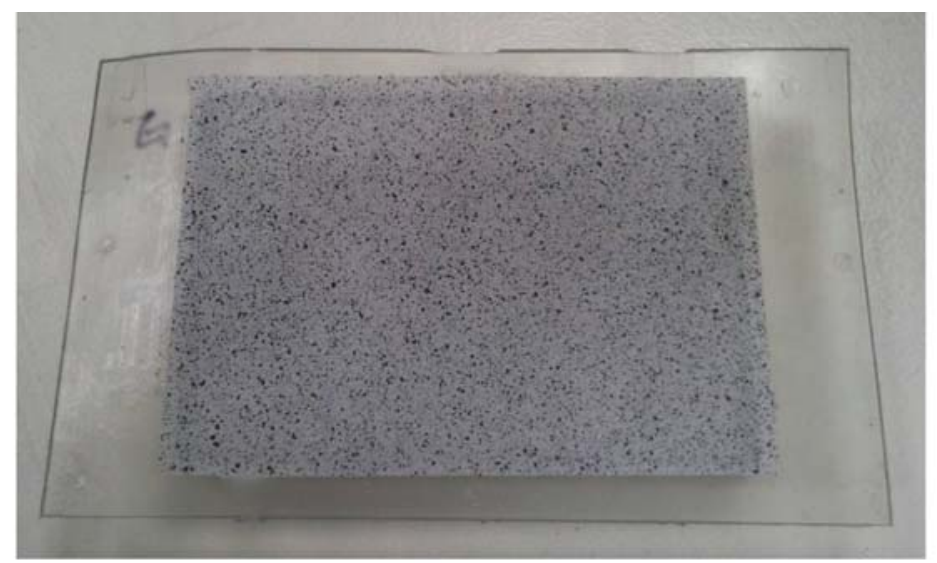

b
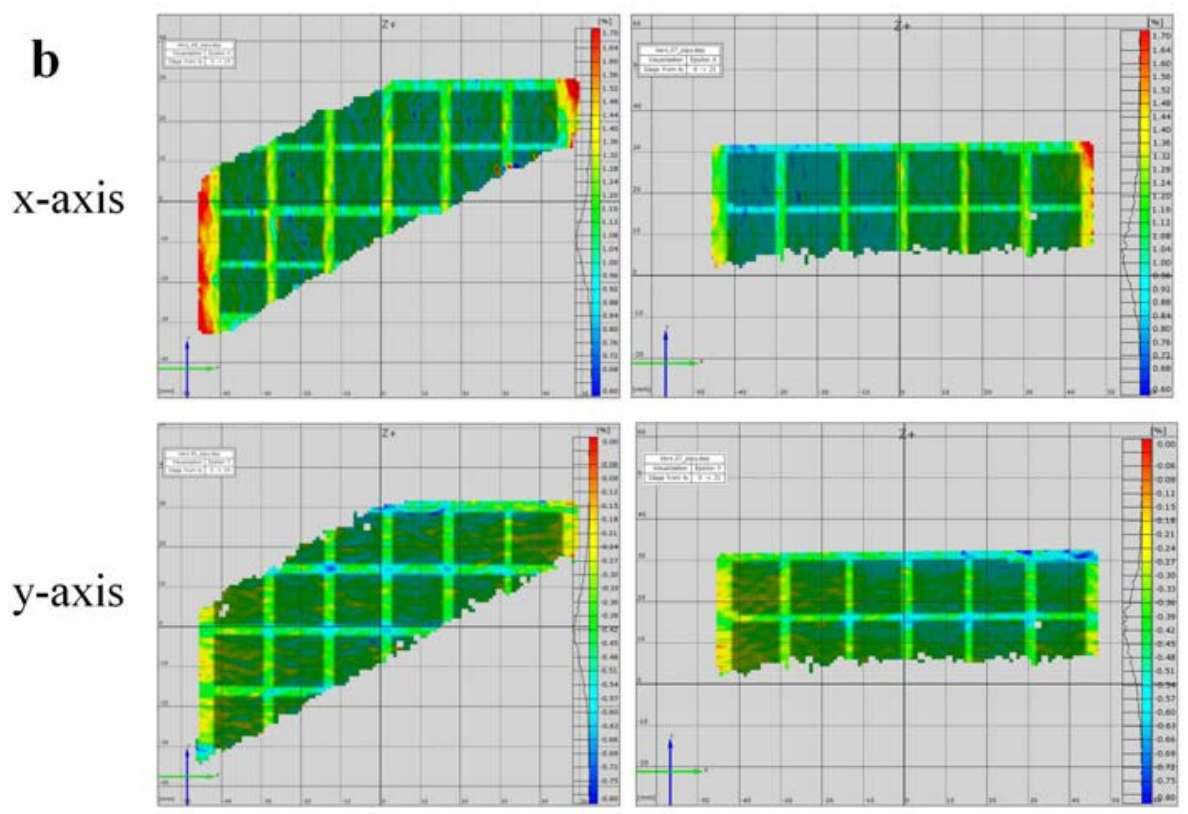

Fig. 3. (a) Stochastic surface pattern applied to the back side of a 24-well PU dish. A representative picture is shown, (b) Optical measurement of strain distribution along the $\mathrm{x}$ - (top) and the y-axis (bottom). Two representative experiments are shown. Identical colors represent equal extension of the PU material after $1 \%$ cyclic strain application.

$\mathrm{Hz}$ and $1 \%(10.000 \mu \varepsilon)$, respectively. Calculations were based on the material properties of elastic polyurethane as basic material for the culture dishes.

For production, the thickness and profile of interior and exterior walls was adapted according to previous FEM calculations. Length of the dishes is $130 \mathrm{~mm}$, width 80 $\mathrm{mm}$, surface area of each single tray is $1.7 \mathrm{~cm}^{2}$ (Fig. 2c). A 3D-CAD engineering drawing of the culture dish was used for production of a rapid prototyping model made of a photoreactive polymer (epoxide resin) by stereolithography (Raprotec, Remscheid, Germany). This model served as archetype to cast a silicon-based female mould, which allows for serial production of the final polyurethane-based dishes by a vacuum casting process. Production was realized with the vacuum casting device (MCP 4/01) and an elastic two-component polyurethane material (both MCP HEK Tooling, Lübeck, Germany; meanwhile MTT Technologies Ltd., Staffordshire, United Kingdom, Data sheets 8020-1, 8020-2) with a final tensile E-modulus of $6 \mathrm{MPa}$, a tensile strength of $8 \mathrm{MPa}$ and a tear strength of $12 \mathrm{MPa}$. By this mode of production, in total 25 cell culture dishes can be produced from one female mould with a maximum deviation caused by production of $0.2 \%$. To prevent microbial colonization, cell cultures dishes were autoclaved before use and protected by a customized lid made of polystyrene.

\section{Optical measurement of strain distribution}

Optical analysis of strain distribution across the culture dishes was accomplished using the System Aramis 2M (GOM, Braunschweig, Germany) with an optical resolution of $1600 \times 1200$ pixels. For visualization of the relative movement at the ground of the culture dishes, a stochastic surface pattern was applied to the back side surface, using a matt white and a graphite spray (Fig. 3a). Measuring on the back side proved to be more accurate, since strain distribution and component characteristics on the front side are identical to those at the back side and there are no dividing walls between the trays that could impair the quality of optical measurements. The two cameras of the Aramis 2M system were calibrated by identifying 13 predefined positions on the calibration plate supplied by the manufacturer. During measurement, the cameras continuously took pictures at an image repeat rate of $12 \mathrm{~Hz}$. To enable exact determination of the maximum strain, image repeat rate has to be 30 times higher than the oscillating frequency. Therefore, cyclic stretching for optical measurement was performed with $1 \%$ strain but 
with at an oscillating frequency of $0.35 \mathrm{~Hz}$. Comprehensive analyses of the pictures allowed for a 3-D view of the surface. All kinds of deformations could be seen as a displacement of the characteristic pattern. Digital image correlation via the Aramis software system allowed for computer-based analysis and quantification of the displacements. Pictures were separately analyzed for each axis ( $\mathrm{x}$ and $\mathrm{y}$ ) of the coordinate plane (Fig. 3b). Statistical analysis comparing independent tests as well as a possible influence of the spatial distribution of individual wells with regard to the 4 different rows and 6 columns of the dishes was performed using analysis of variance (ANOVA).

\section{Cell culture}

Media for cell culture and FCS were obtained from PAA Laboratories, Linz, Austria. Telomerase immortalized human mesenchymal stem cells (hMSC-TERT) were cultivated as described (Simonsen et al., 2002) in Earle's MEM containing $10 \%$ FCS. Primary human mesenchymal stem cells (hMSC) were isolated from bone marrow from different donors and cultivated by a standardized protocol (Noth et al., 2002). Bone marrows were recovered after informed consent from the explanted femoral heads of patients undergoing elective hip arthroplasty. The procedure was approved by the local Ethics Committee of the University of Würzburg. Briefly, bone marrow preparations were washed with Dulbecco's modified Eagle's medium, (DMEM/F12) supplemented with 10\% FCS, $100 \mathrm{U} / \mathrm{ml}$ penicillin, $0.1 \mathrm{mg} / \mathrm{ml}$ streptomycin, and $50 \mu \mathrm{g} / \mathrm{ml}$ ascorbate (Sigma-Aldrich, München, Germany), and centrifuged at $1200 \mathrm{rpm}$ for $5 \mathrm{~min}$. The pellet was reconstituted in medium and washed four times, and the supernatants of the washing steps containing the released cells were collected. Cells were centrifuged and cultivated at a density of $3 \times 10^{8}$ cells per $150 \mathrm{~cm}^{2}$ culture flask. Adherent cells were washed after 2 days and cultivated until confluence. HEK293 cells (ATCC No. CRL-1573) were grown in Dulbecco's modified Eagle's medium (DMEM high glucose) containing $10 \%$ FCS. All cells were grown at $37^{\circ} \mathrm{C}$ in a humidified atmosphere consisting of 5 $\% \mathrm{CO}_{2}$ and $95 \%$ air.

\section{Cloning of AP1 and SP1 responsive constructs}

For functional analyses oligonucleotides containing AP1 and SP1 responsive elements (AP1: 5'-ATC TGA CTC AGC ATG CAT GTG ACT CAG CTA-3'; SP1: 5'-ATC GCG GCG GGG GCG GGC GCC GCA TGC ATG GCG GCG GGG GCG GGC GCC GCT A-3') were cloned in between the EcoRI sites of the pCRII vector (Invitrogen GmbH, Karlsruhe, Germany). Both, the AP1 and the SP1 sites were subcloned into the luciferase reporter vector pGL4.14[luc2/Hygro] (Promega, Mannheim, Germany) via SacI and XhoI restriction (all restriction endonucleases from New England Biolabs, Frankfurt am Main, Germany) to receive the pGL4.14$\mathrm{SP} 1$ vector and via $\mathrm{XhoI}$ and $\mathrm{BamHI}$ restriction to receive the pGL4.14-AP1 vector and sequenced with the RVprimer3 (Promega) by dye terminator sequencing using the ABI 310 capillary sequencer as described previously. Constructs were used for transient transfection of HEK293 cells and for establishing stable hMSC-TERT cells lines.

\section{Establishing stable hMSC-TERT-AP1 and hMSC- TERT-SP1 clones}

The vectors pGL4.14-AP1, pGL4.14-SP1 and pGL4.14[luc2/Hygro] were linearised with SacII. 1 x $10^{6}$ hMSC-TERT cells were mixed with $10 \mu \mathrm{g}$ DNA in $400 \mu \mathrm{l}$ serum-free medium in $2 \mathrm{~mm}$ wide cuvettes (Bio-Rad Laboratories, München, Germany) and transfected by electroporation at $120 \mathrm{~V}$ and $950 \mu \mathrm{F}$ using a Gene Pulser 2 (Bio-Rad Laboratories $\mathrm{GmbH}$ ). Cells were seeded into two wells of a 6-well plate and cultured overnight in serumcontaining medium. $24 \mathrm{~h}$ post transfection $50 \mu \mathrm{g} / \mathrm{ml}$ hygromycin was added to select positive hMSC-TERTAP1, hMSC-TERT-SP1 and hMSC-TERT clones containing the empty pGL4.14[1uc2/Hygro] vector (hMSC-TERT-control). For cyclic stretching $3 \times 10^{4}$ cells were seeded per well on 24-well PU plates and cultivated for $48 \mathrm{~h}$.

To control stabile integration genomic DNA was isolated from stable hMSC-TERT cells using the QiAamp DNA Mini Kit (Qiagen, Hilden, Germany) according to the manufacturer's instructions. PCR was performed with 50 ng genomic DNA using a PTC-200 Peltier thermal cycler (Bio-Rad Laboratories) in a volume of $50 \mu \mathrm{l}$ (forward primer: RVprimer3 localized from base 5790 to base 5809; reverse primer 5'-TCATGGCTTTGTGC AGCTGC-3', localized from base 171 to base 190 of the pGL4.14[luc2/Hygro] vector). Primer oligonucleotides were purchased from Biomers (Ulm, Germany). Genomic DNA was amplified with Taq-polymerase purchased from Peqlab Biotechnologie (Erlangen, Germany) PCR bands were sequenced with the RVprimer3 as described above.

\section{Cloning of the lentiviral luciferase reporter construct} As no suitable luciferase reporter vector for lentiviral transduction of primary cells was available on the market, we had to establish a construct, which fulfils our needs. First of all the SP1 and the AP1 responsive elements were cloned into $\mathrm{pENTR}^{\mathrm{TM}} 5^{6}$-TOPO, respectively, the coding sequence of the firefly luciferase was cloned into the KpnI and NotI sites of the pENTR ${ }^{\mathrm{TM}} 11$ vector. The empty pENTR $^{\mathrm{TM}} 5^{6}$-TOPO vector was used for generating the control plasmid. The $\mathrm{pENTR}^{\mathrm{TM}} 11$-luciferase and the pENTR ${ }^{\mathrm{TM}} 5^{6}-\mathrm{TOPO}-\mathrm{SP} 1, \mathrm{pENTR}{ }^{\mathrm{TM}} 5^{6}-\mathrm{TOPO}-\mathrm{AP} 1$ or the pENTR $^{\mathrm{TM}} 5$ ‘-TOPO construct, respectively were recombined with the destination vector $\mathrm{pLenti6/R4R2/V5-}$ Dest (all vectors from Invitrogen, Karlsruhe, Germany) according to the manufacturer's instructions to receive the lentiviral luciferase SP1 reporter vector pLenti-SP1, the lentiviral luciferase AP1 reporter vector pLenti-AP1 and the control vector pLenti- 0 , which contains the luciferase coding sequence but no mechanoresponsive element.

\section{Transient transfection of HEK293 cells}

For transient transfection, HEK293 cells were seeded in the 24-well PU dishes $\left(1.8 \times 10^{4}\right.$ cells/well $)$ and transfected at $50-70 \%$ confluence. Liposome-mediated transfection was performed using LipofectAMINE reagent (Invitrogen). $2 \mu \mathrm{l}$ of LipofectAMINE and $0.9 \mu \mathrm{g}$ of the pGL4.14-AP1 reporter construct and the empty pGL4.14[luc2/Hygro] vector, respectively were diluted in $100 \mu \mathrm{l}$ serum-free medium and incubated for $30 \mathrm{~min} .124$ 
$\mu \mathrm{l}$ serum-free medium was added, and the solution was then carefully dripped onto the cells. HEK293 cells were incubated for $5 \mathrm{~h}$ in transfection solution. After removal of the medium cells were cultivated with serum-containing medium. After $24 \mathrm{~h}$ the cells were stimulated by cyclic stretching.

\section{Lentiviral transduction of primary mesenchymal stem cells}

After cotransfection of the lentiviral reporter constructs pLenti-SP1, pLenti-AP1 and pLenti-0 into 293FT cells by using the ViraPower ${ }^{\mathrm{TM}}$ Packaging Mix (all Invitrogen), 5 $\mathrm{ml}$ supernatant was mixed with $10 \mathrm{ml}$ basal medium and used to transduce primary hMSC seeded in $75 \mathrm{~cm}^{2}$ culture flasks at $50 \%$ confluency. After 4 days $2 \mu \mathrm{g} / \mathrm{ml}$ blasticidin was added to select stably transduced cells. After 5 medium changes $(2-3$ weeks) cells were detached from the culture flasks, counted and $3 \times 10^{4}$ cells $/ 1.7 \mathrm{~cm}^{2}$ well were seeded on the flexible PU dishes.

\section{Cyclic stretching of HEK293, hMSC-TERT and} primary mesenchymal stem cells and luciferase assay Cyclic stretching was performed for $30 \mathrm{~min}$ at 0.05 to 2 $\mathrm{Hz}$ and $0.5 \%$ to $5 \%$ extension. To analyze AP1 signaling specificity, hMSC-TERT-AP1 cells were pre-treated before stretching for 2 days with or without $5 \mu \mathrm{M}$ verapamil (Sigma Aldrich, Munich, Germany), an calcium channel blocker. After stretching and cultivating the cells for another $24 \mathrm{~h}$, cells were harvested and lysed in $150 \mu \mathrm{l}$ Reporter Lysis Buffer (Promega). $20 \mu \mathrm{l}$ of each extract was analyzed for luciferase activity using the reporter gene assay provided by Promega $\mathrm{GmbH}$ in an Orion II Luminometer (Berthold Detection Systems, Pforzheim, Germany) in 96-well plates. Relative light units were normalized to protein content determined by using RotiQuant Protein Assay (Carl Roth, Karlsruhe, Germany) and the data from four wells were used to calculate the mean value.

\section{Proliferation assay}

$3 \times 10^{4}$ hMSC-TERT-AP1 cells / well were seeded on the newly developed 24-well PU dishes and on conventional PS 24-well plates and the proliferation ratio was analyzed by using the WST-1 cell proliferation reagent (Roche Diagnostics, Mannheim, Germany) according to the manufacturer's instructions. Briefly, $100 \mu \mathrm{lWST}-1$ reagent was added after 1, 3 and 6 days to $1 \mathrm{ml}$ culture medium and incubated for $30 \mathrm{~min}$ in a cell incubator. $100 \mu \mathrm{l}$ were transferred to a 96-well plate and absorption was analyzed at $450 \mathrm{~nm}$ (reference wavelength $620 \mathrm{~nm}$ ) with a Tecan sunrise microplate reader and the Magellan V6.6 software (Tecan Group, Männedorf, Switzerland). Experiments were repeated three times and were performed in triplicates.

\section{RT-PCR analysis of mechanoresponsive HB-GAM}

HMSCs cells were seeded on PU dishes in a density of $3 \mathrm{x}$ $10^{4}$ cells $/ \mathrm{cm}^{2}$ and cyclic stretched for $30 \mathrm{~min}$ at $1 \mathrm{~Hz}$ and $1 \%$ extension. Total RNA was immediately isolated using the NucleoSpin RNA II kit (Macherey-Nagel, Düren, Germany) according to the manufacturer's instructions.
Two micrograms of total RNA were reverse-transcribed with MMLV reverse transcriptase (Promega $\mathrm{GmbH}$ ) in a volume of $20 \mu \mathrm{l}$. For amplification of mechanoresponsive HB-GAM (primers HB-GAM sense (5' GCAAACCATGAAGACCCAGA-3'), HB-GAM antisense (5'-GGCTTGGAGATGGTGACAGT-3'), annealing temperature $60^{\circ} \mathrm{C}$ ) (Neidlinger-Wilke et al., 2009) $1 \mu \mathrm{l}$ of cDNA was used as a template in a volume of $50 \mu 1$. Taq DNA polymerase was obtained from Peqlab (Erlangen, Germany). PCR conditions were as follows: 30 seconds at $94^{\circ} \mathrm{C}, 30$ seconds at annealing temperature, 60 seconds at $72^{\circ} \mathrm{C}$. As a housekeeping gene EF1a was amplified (primers: EF1a sense (5'-AGGTGATTATC CTGAACCATCC-3'), EF1a antisense (5'-AAAGG TGGATAGTCTGAGAAG-3'), annealing temperature $54^{\circ} \mathrm{C}$ ). Experiments were performed with three different preparations of primary hMSC. PCR bands were semiquantitatively analyzed by densitometry measurements using the Bio1D software (Vilber Lourmat, Eberhardzell, Germany).

\section{Membrane staining with concanavalin A}

HMSC-TERT-AP1 cells $\left(3 \times 10^{4}\right.$ cells $\left./ \mathrm{cm}^{2}\right)$ were cultivated for 3 days on PU dishes. Then, the cells were fixed with $4 \%$ PFA and permeabilized with $0.2 \%$ Triton X-100. Membrane-staining dye, $15 \mu \mathrm{g} / \mathrm{ml}$ concanavalin A-Texas Red (Invitrogen $\mathrm{GmbH}$ ) was applied in PBS for 30 min at $4^{\circ} \mathrm{C}$. After washing with PBS, nuclear counter staining with DAPI (Invitrogen) was performed for $5 \mathrm{~min}$ at room temperature. Cells were photomicrographed with an AxioCam MRm camera on an Axiovert S100 microscope (Carl Zeiss). Three independent stainings were performed.

\section{Results}

\section{Finite element analysis of polyurethane cell culture dishes}

Different types of 24-well culture dishes regarding shape and assembly of the individual trays were planed and strain distribution analyzed by FEM calculation (Fig. 2a). Eventually, a construction of 24 contiguous, rectangular wells with selective modifications of the thickness and shape of distinct partition walls in conjunction with a chucking device covering the full breadth of the dishes proved to provide best possible approximation to a largely homogenous strain distribution within each tray as well as between the trays at different positions on the 24-well culture dish (Fig. 2a and b).

\section{Optical measurement of strain distribution}

Strain values and there spatial distribution throughout the PU culture dishes were measured optically employing the complete bioreactor system as used for cell culture experiments to allow for a comprehensive analysis of the actual strain values comprising all potential error sources. Only those areas of the dish, where the fineness of the stochastic pattern was sufficient to allow valid analysis were used for calculation of strain distribution (Fig. 3b). In order to allow unbiased evaluation, no technical measures for exponential smoothening were applied so 
a

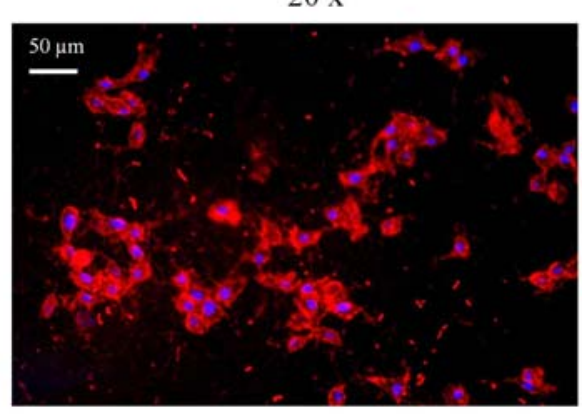

b

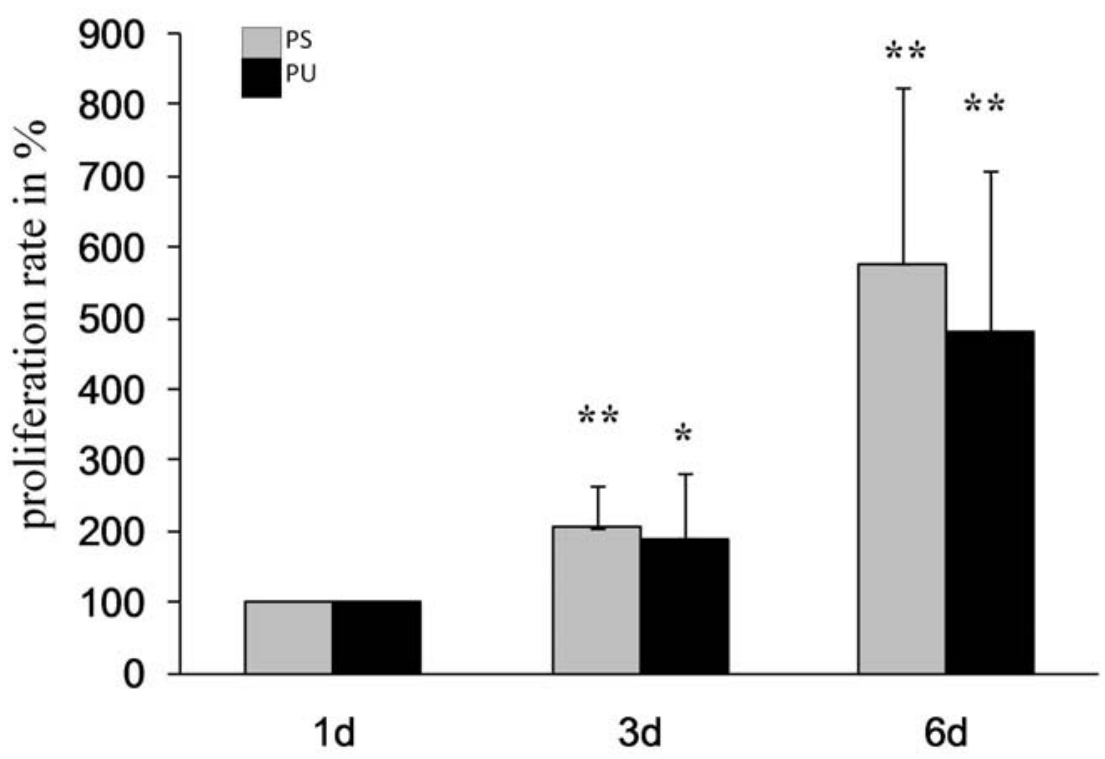

$40 x$

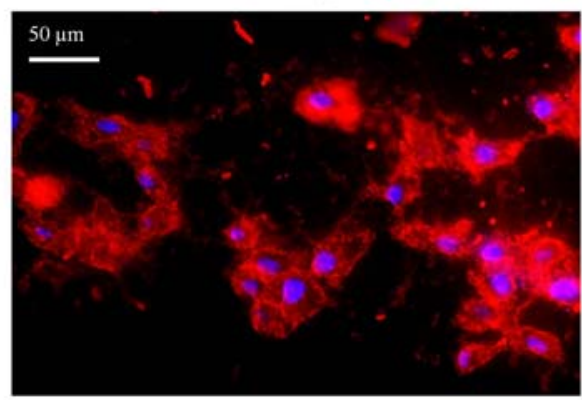

Fig. 4. (a) hMSC-TERTAP1 cells grown on PU dishes and stained with concanavalin A (red) and counterstained with DAPI (blue). The bar represents 50 $\mu \mathrm{m}$. (b) Proliferation rate of stable hMSC-TERT-AP1 cells grown on conventional polystyrene (PS, grey bars) and on polyurethane (PU, black bars) dishes for 1, 3 and 6 days without any mechanical stimulation. The data were obtained from three measure points from three independent experiments and are shown as means \pm SEM. Statistics were calculated with the Student's t-test $(* *: p<0.001$, $*: p<0.01)$. areas, where the stochastic pattern was too rough were strictly excluded. In single experiments, only partial aspects of the graphite spray pattern met these technical requirements and gave valid results. As described in the materials and methods section, the strain applied along the $\mathrm{x}$-axis was $1 \%$. Evaluation was focused on single trays. In total five independent experiments comprising 52 individual wells with 84 to 256 (average 207.6) measuring points per well were evaluated. The average strains of the single wells varied between $0.907 \%$ and $1.132 \%$ with the average value of the means being $1.043 \%$. Standard deviation of the means was calculated as $0.047 \%$. Mean standard deviation was $0.090 \%$ (min $0.059 \%$, max 0.147 $\%)$. The minimum-values of all wells analyzed varied between $0.645 \%$ and $0.945 \%$, respectively with a mean minimum at $0.791 \%$. Regarding all trays, average maximum was $1.32 \%(\min 1.146 \%$, $\max 1.524 \%)$. The average range between maximum and minimum was 0.528 $\%$. Analysis of variance of the independent experiments showed no significant difference with $p=0.35$ and $F=$ 1.13 (critical value 2.57). Statistical comparison (ANOVA) of the arithmetic means of the four different rows ( $p=$ $0.26 ; F=1.37$, critical value 2.80 ) and the six columns ( $p$ $=0.87 ; F=0.87$, critical value 2.42 ) also did not show any significant differences. Accordingly, visual analysis of the pattern of strain distribution did not show any significant pattern of areas with increased or alleviated strain. Deformation along the y-axis perpendicular to the intended loading direction is transverse contraction, thus strain values measured were negative. The arithmetic mean of transverse contraction over all wells was $-0.416 \%$. Mean values of the individual wells ranged from $-0.328 \%$ to $0.474 \%$. Again, no obvious pattern of inhomogeneous strain distribution was observed and statistical comparison (ANOVA) of the arithmetic means of the four different rows $(p=0.16 ; F=1.79$; critical value 2.80$)$ and the six columns $(p=0.48 ; F=0.91$, critical value 2.42$)$ also did not show any significant differences. Thus strain can be supposed to be independent of the spatial distribution of the wells on the culture dish.

\section{Cell growth and adhesion characteristics of cells in PU dishes}

Since the PU material is not translucent and did not allow for direct microscopic control of cell cultures, populations of cells were stained with concanavalin A and counterstained with DAPI. Sufficient growth and spreading was observed during day 1-6 (Fig. 4a) until after seven days in culture the cell layer started to detach. In consequence, any stretching experiment was designed for culture in PU dishes of less than 7 days and the number of cells seeded was $3 \times 10^{4}$ / well to achieve sufficient cellcell contacts and to get the cells as closely as possible to confluence by the start of stretching experiments. To exclude any influence of the PU material on cellular growth, hMSC-TERT-AP1 cells were seeded on 
a

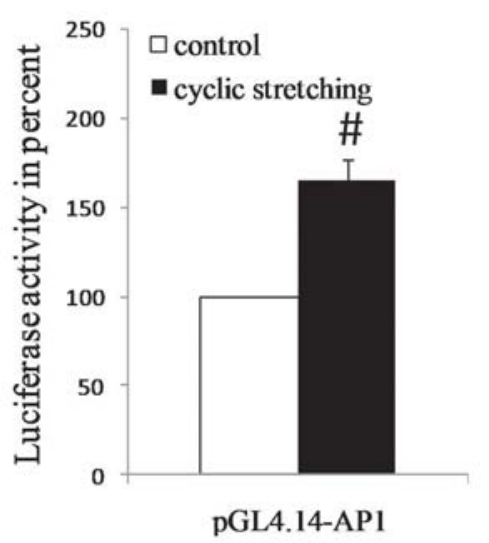

b

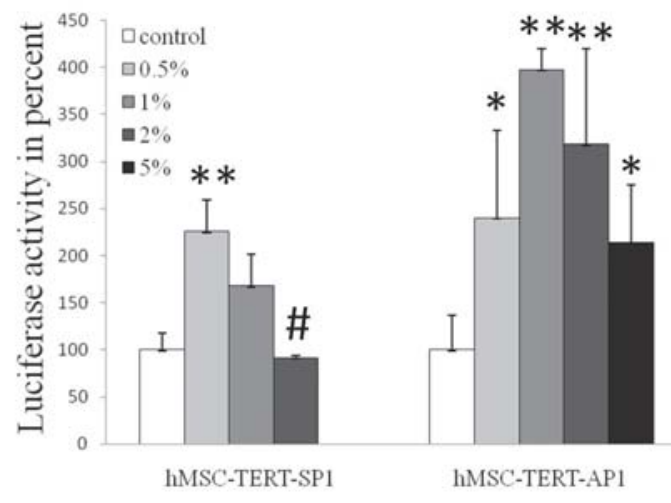

d

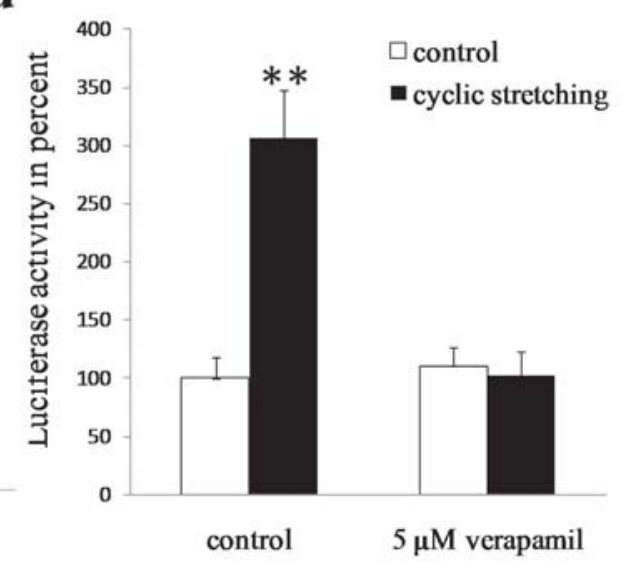

Fig. 5. Luciferase activity of strain responsive reporter constructs. (a) Transient transfection of the pGL4.14-AP1 reporter construct into HEK293 cells and stimulation of cells by cyclic stretching with $1 \%$ extension and $1 \mathrm{~Hz}$ (black bar). The white bar represents the unstimulated control. (b) Stable hMSC-TERT-AP1 and hMSC-SP1 cells were stimulated with variable extensions but a constant frequency of $1 \mathrm{~Hz}$. (c) hMSC-TERT-AP1 cells were stimulated with variable frequencies but a constant extension of $1 \%$. (d) hMSC-TERT-AP1 cells were treated with the calcium channel blocker verapamil and stimulated by cyclic stretching with $1 \%$ extension and $1 \mathrm{~Hz}$ (black bars). The white bars represent the non-stretched control. All data were obtained from three independent experiments with four measure points each and are shown as means \pm SEM. Statistics were calculated with the Student's $t$-test $(* *$ : $p<0.001, *: p<0.01, *: p<0.05)$.

conventional PS 24-well plates and on the here described 24-well PU dishes and proliferation was determined with the WST-1 assay after 1, 3 and 6 days in comparison without any mechanical stimulations (Fig. 4b). Proliferation increased in cells grown on either surface, PS (grey bars) or PU (black bars), from day 1 to day 6 , which excluded any unfavorable impact of the PU material on cellular viability and growth although the proliferation rate was lower on PU compared to PS after 6 days.

\section{Establishing of mechanoresponsive AP1 luciferase reporter constructs}

To test if the newly established PU dishes are suitable for applying mechanical strain to cells and if the strain responsive luciferase reporter constructs are responding to mechanical stimuli HEK293 cells were exemplarily transiently transfected with the pGL4.14-AP1 construct and the pGL4.14[luc2/Hygro] vector as a control. After cyclic stretching luciferase activity was induced nearly 2fold in pGL4.14-AP1 transfected cells (Fig. 5a, black bar) compared to control cells (white bar). The pGL4.14[luc2/ Hygro] transfected control cells did not respond to cyclic stretching (data not shown).

\section{Characterization of stable mechanoresponsive AP1} and SP1 cell lines

After we received the proof-of-principle that the construct as well as the PU dishes are suitable for analyzing mechanical strain applied to cell monolayers we established stable hMSC-TERT-AP1 and hMSC-TERT-SP1 cells, which were stimulated with variable extensions from $0.5 \%$ to $2 \%$ (SP1 clone) and $0.5 \%$ to $5 \%$ (AP1 clone), respectively. The SP1 construct showed the highest responsiveness at an extension of $0.5 \%$, a declining activity at $1 \%$ extension and no effect at $2 \%$ strain (Fig. 5b, left panel), while the AP1 construct was most responsive at $1 \%$ extension and showed a lower induction when amplitudes of $0.5 \%, 2 \%$ and $5 \%$ were applied (Fig. 5b, right panel). The hMSC-TERT-control cells were not responsive to mechanical strain (data not shown).

Additionally, different frequencies starting from 0.05 $\mathrm{Hz}$ to $2 \mathrm{~Hz}$ were applied to hMSC-TERT-AP1 cells and luciferase activity was determined. The AP1 responsive element was strongly induced at $0.05 \mathrm{~Hz}$ and $1 \mathrm{~Hz}$, while other applied frequencies displayed a lower induction of luciferase activity (Fig. 5c). To validate the specificity of AP1 signaling, cells were pre-treated for 2 days with the 

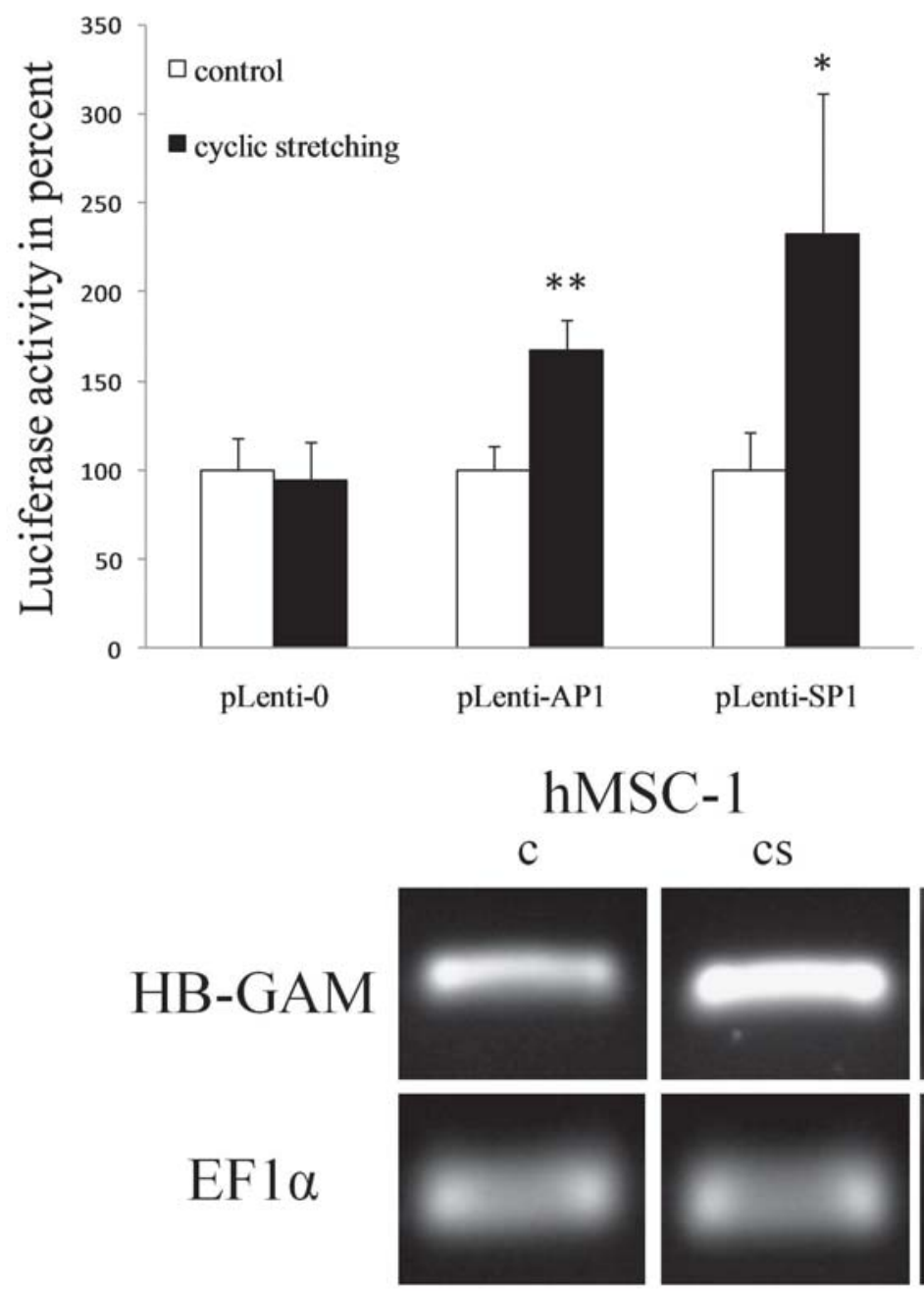

Fig. 6. Lentiviral transduction of primary mesenchymal stem cells with the newly developed luciferase reporter vectors. pLenti-AP1, pLenti-SP1 and pLenti-0 luciferase constructs were transduced into hMSC and cells were stimulated by $1 \%$ and $1 \mathrm{~Hz}$ cyclic stretching (black bars). White bars represent unstimulated controls. The data were obtained from 4 different measure points from four independent experiments and are shown as means \pm SEM. Statistics were calculated with the Student's $t$-test $(* *$ : $p<0.001, *: p<0.01)$.

\section{hMSC-2}

c
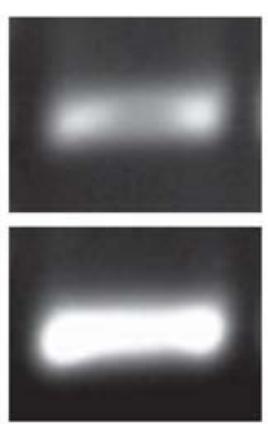

CS

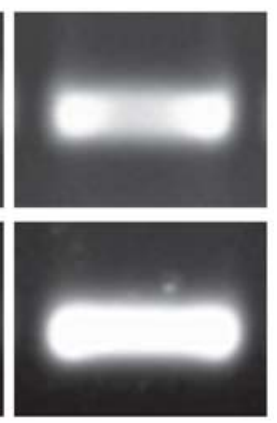

Fig. 7. Expression of the mechanoresponsive gene HB-GAM in hMSC after cyclic stretching. As a housekeeping gene EF $1 \alpha$ was amplified. Two representative experiments are shown.

calcium channel blocker verapamil, which completely abolished the AP1 mediated mechanoresponsivity in hMSC-TERT-AP1 cells (Fig. 5d). No effect of verapamil was seen in hMSC-TERT control cells (data not shown).

\section{Mechanosensitivity of primary human mesenchymal stem cells}

The coding sequence of the firefly luciferase, the SP1 and the AP1 responsive elements were cloned into a lentiviral expression vector ( $\mathrm{pLenti-SP1}$ and pLenti-AP1) to have the opportunity to analyze mechanical strain in primary mesenchymal stem cells. After applying mechanical stimuli to $\mathrm{pLenti-AP} 1$ and $\mathrm{pLenti-SP} 1$ transduced primary hMSC luciferase activity was enhanced 1.6-fold and 2.3-fold, respectively, while cells transduced with the empty vector pLenti-0 did not respond to mechanical strain (Fig. 5).

Regulation of the mechanoresponsive gene HB-GAM To further analyze if the here described bioreactor and the PU dishes are suitable for mechanical stimulation three different preparations of primary mesenchymal stem cells were stimulated with cyclic stretching and the expression of the mechanoresponsive gene HB-GAM was analyzed by RT-PCR in three different hMSC preparations. Bands were densitometrically evaluated. The expression of HBGAM was significantly upregulated 1.7-fold ( $\mathrm{SEM} \pm 0.24$; $p<0.05)$ by cyclic stretching although a high donor variability was observed (Fig. 7).

\section{Discussion}

In this work we describe a newly developed bioreactor suitable for applying 2-D mechanical strain to cellular monolayers grown in small scale formats on polyurethane surfaces. A variety of bioreactor systems capable of applying mechanical strain to 2-D and 3-D cell cultures were developed and are reviewed by Brown (Brown, 2000). Mechanical strain can be applied to cells by fluid shear stress (Wang et al., 2010), by using compression systems (Bougault et al., 2008) or by bending circular substrates (Cha and Purslow, 2010). One commercially available cellular strain device (FX-4000 T, Flexcell International Corporation, Hillsborough, NC, USA) was extensively characterized by the group of G. Duda (Bieler et al., 2009) in terms of strain distribution across the surface 
of the culture dishes by digital image correlation techniques (Cottrell et al., 2009). The authors showed that only cells adhering to a central area $1.8 \mathrm{~cm}$ in diameter are subjected to homogenous mechanical strain while cells surrounding this area receive higher strain extensions. It would result in misleading impressions in terms of mechanically induced effects if cells were pooled across the whole well area. These findings demonstrate that there is a need for cell culture dishes, which have a homogenous strain distribution across the whole well surface when subjected to mechanical strain. Our newly developed PU dishes are intended to fulfill this criterion as we demonstrated by finite element analysis and optical measurements. We have previously described and applied a reactor system using $18 \mathrm{~cm}^{2}$ silicone dishes (Liedert et al., 2006a; Liedert et al., 2009), which we now adapted for small scale applications. Moreover our 24-well format well-design combined with the sensitive luciferase readout allows for small volume analyses with comparably high throughput, which is of specific importance when precious primary cell cultures are analyzed. Additionally, the 24-well format of our PU dishes allows for stimulating multiple samples simultaneously, which is beneficial in terms of identical culture and stimulation conditions. Cells showed reasonable adherence to the newly developed PU dishes demonstrated by concanavalin A staining and proliferation rates comparable to rates obtained from cells grown on conventional PS.

The newly developed reactor and the PU dishes are suitable for stimulating primary cells and cell lines with mechanical load. In order to get a sensitive and reliable readout for the effects of strain application we tested a self-established reporter gene assay comprising known mechanosensitive response elements, which control luciferase expression. By using these luciferase-based mechanoresponsive reporter constructs we could demonstrate that AP1 and SP1 responsive elements were induced by cyclic strain applied to stable hMSC-TERT cells and cyclic strain induced lentivirally transduced SP1 and AP1 responsive elements in primary hMSC. The specificity of the AP1 activation was analyzed by cotreating stretched hMSC-TERT-AP1 cells with verapamil, a calcium channel blocker, which disrupts the characteristic signaling cascade initialized from the interaction of mechanical forces with membrane-associated proteins resulting in calcium influx, ERK1/2 activation and transcriptional induction of e.g. c-fos proteins (Li et al., 2002). As previously demonstrated the promoter of the mechanosensitive gene HB-GAM comprises an AP1 site, which probably mediates mechanotransduction (Liedert et al., 2009).

The established hMSC-TERT-AP1 and hMSC-TERTSP1 cell lines are a suitable additional tool and screening system for testing putative mechanosensitizers / inhibitors of mechanotransduction in a small scale format. As hMSCTERT cells are mesenchymal stem cells immortalized with telomerase they are a stable and represent a versatile system for the here described screening procedures. They can be used over a long time period without the risk of proliferation retardation or spontaneous differentiation induction. As we could previously show they can be differentiated into osteogenic, adipogenic and chondrogenic precursors to a certain extend to scan also differential mechanotransduction-related effects along mesenchymal differentiation pathways (Abdallah et al., 2005).

After we adduced the proof-of-principle that our established luciferase reporter constructs respond do mechanical load, we cloned the responsive elements together with the coding sequence for firefly luciferase into a lentiviral vector system to have the opportunity to transduce primary cells for maximal flexibility. We could show that the constructs are suitable for investigating mechanical load on primary cells and provide a versatile tool for analyzing mechanical effects on cells isolated from patients with impaired mechanotransduction, e.g. MSC from osteoporotic subjects.

In summary we describe a complete and comprehensive solution for a small scale cell culture/bioreactor system including sensitive readout systems and clonal cell populations capable of analyzing subcellular crosstalk mechanisms in mechanotransduction, mechanosensitivity of primary cells and of screening the activity of putative mechanosensitizers as new targets. This will be relevant for the treatment of e.g. bone loss caused by both disuse and signal transduction related alterations of mechanotransduction in degenerative diseases.

\section{Acknowledgements}

This work was supported by grants from the German Research Foundation (FOR793, JA504/10-1), the Bavarian Research Foundation (ForZebRA) the German Society for Orthopedics and Orthopedic Surgery (DGOOC) and the EXIST program of the Federal Ministry of Economics and Technology. We thank Martina Burggraf for excellent technical assistance.

\section{References}

Abdallah BM, Haack-Sorensen M, Burns JS, Elsnab B, Jakob F, Hokland P, Kassem M (2005) Maintenance of differentiation potential of human bone marrow mesenchymal stem cells immortalized by human telomerase reverse transcriptase gene despite [corrected] extensive proliferation. Biochem Biophys Res Commun 326: 527-538.

Armstrong VJ, Muzylak M, Sunters A, Zaman G., Saxon LK, Price JS, Lanyon LE (2007) Wnt/beta-catenin signaling is a component of osteoblastic bone cell early responses to load-bearing and requires estrogen receptor alpha. J Biol Chem 282: 20715-20727.

Asparuhova MB, Gelman L, Chiquet M (2009) Role of the actin cytoskeleton in tuning cellular responses to external mechanical stress. Scand J Med Sci Sports 19: 490-499.

Bieler FH., Ott CE, Thompson MS, Seidel R, Ahrens S, Epari DR, Wilkening U, Schaser KD, Mundlos S, Duda GN (2009) Biaxial cell stimulation: A mechanical validation. J Biomech 42: 1692-1696. 
Bougault C, Paumier A, Aubert-Foucher E, MalleinGerin F (2008) Molecular analysis of chondrocytes cultured in agarose in response to dynamic compression. BMC Biotechnol 8: 71.

Brown TD (2000) Techniques for mechanical stimulation of cells in vitro: a review. J Biomech 33: 3-14. Cha MC, Purslow PP (2010) The activities of MMP-9 and total gelatinase respond differently to substrate coating and cyclic mechanical stretching in fibroblasts and myoblasts. Cell Biol Int 34: 587-591.

Cottrell JA, Meyenhofer M, Medicherla S, Higgins L, O’Connor JP (2009) Analgesic effects of p38 kinase inhibitor treatment on bone fracture healing. Pain 142: 116126.

Hahn C, Schwartz MA (2009) Mechanotransduction in vascular physiology and atherogenesis. Nat Rev Mol Cell Biol 10: 53-62.

Huveneers S, Danen EH (2009) Adhesion signaling crosstalk between integrins, Src and Rho. J Cell Sci 122: 1059-1069.

Inoue D, Kido S, Matsumoto T (2004) Transcriptional induction of FosB/DeltaFosB gene by mechanical stress in osteoblasts. J Biol Chem 279: 49795-49803.

Jani K, Schock F (2009) Molecular mechanisms of mechanosensing in muscle development. Dev Dyn 238: 1526-1534.

Judex S, Gupta S, Rubin C (2009) Regulation of mechanical signals in bone. Orthod Craniofac Res 12: 94 104.

Khatiwala CB, Kim PD, Peyton SR, Putnam AJ (2009) ECM compliance regulates osteogenesis by influencing MAPK signaling downstream of RhoA and ROCK. J Bone Miner Res 24: 886-898.

Kiselyov K, Patterson RL (2009) The integrative function of TRPC channels. Front Biosci 14: 45-58.

Kletsas D, Basdra EK, Papavassiliou AG. (2002) Effect of protein kinase inhibitors on the stretch-elicited c-Fos and c-Jun up-regulation in human PDL osteoblast-like cells. J Cell Physiol 190: 313-321.

Klossner S, Durieux AC, Freyssenet D, Flueck M (2009) Mechano-transduction to muscle protein synthesis is modulated by FAK. Eur J Appl Physiol 106: 389-398.

Li J, Duncan RL, Burr DB, Turner CH (2002) L-type calcium channels mediate mechanically induced bone formation in vivo. J Bone Miner Res 17: 1795-1800.

Liedert A, Kaspar D, Blakytny R, Claes L, Ignatius A (2006a) Signal transduction pathways involved in mechanotransduction in bone cells. Biochem Biophys Res Commun 349: 1-5.

Liedert A, Kaspar D, Claes L, Ignatius A (2006b) Signal transduction pathways involved in mechanical regulation of HB-GAM expression in osteoblastic cells. Biochem Biophys Res Commun 342: 1070-1076.

Liedert A, Kassem M, Claes L, Ignatius A (2009) Mechanosensitive promoter region in the human HB-GAM gene. Biochem Biophys Res Commun 387: 289-293.

Liedert A, Wagner L, Seefried L, Ebert R, Jakob F, Ignatius A (2010). Estrogen receptor and Wnt signaling interact to regulate early gene expression in response to mechanical strain in osteoblastic cells. Biochem Biophys Res Commun 394: 755-759.

Liu J, Zhao Z, Li J, Zou L, Shuler C, Zou Y, Huang X, Li M, Wang J (2009). Hydrostatic pressures promote initial osteodifferentiation with ERK1/2 not p38 MAPK signaling involved. J Cell Biochem 107: 224-232.

Miyagi M, Miwa Y, Takahashi-Yanaga F, Morimoto S, Sasaguri T (2005) Activator protein-1 mediates shear stress-induced prostaglandin d synthase gene expression in vascular endothelial cells. Arterioscler Thromb Vasc Biol 25: 970-975.

Neidlinger-Wilke C, Liedert A, Wuertz K, Buser Z, Rinkler C, Kafer W, Ignatius A, Claes L, Roberts S, Johnson WE (2009) Mechanical stimulation alters pleiotrophin and aggrecan expression by human intervertebral disc cells and influences their capacity to stimulate endothelial migration. Spine 34: 663-669.

Noth U, Osyczka AM, Tuli R, Hickok NJ, Danielson KG, Tuan RS (2002) Multilineage mesenchymal differentiation potential of human trabecular bone-derived cells. J Orthop Res 20: 1060-1069.

Ogasawara A, Arakawa T, Kaneda T, Takuma T, Sato T, Kaneko H, Kumegawa M, Hakeda Y (2001) Fluid shear stress-induced cyclooxygenase- 2 expression is mediated by C/EBP beta, cAMP-response element-binding protein, and AP-1 in osteoblastic MC3T3-E1 cells. J Biol Chem 276: 7048-7054.

Papachroni KK, Karatzas DN, Papavassiliou KA, Basdra EK, Papavassiliou AG (2009) Mechanotransduction in osteoblast regulation and bone disease. Trends Mol Med 15: 208-216.

Peverali FA, Basdra EK, Papavassiliou AG (2001) Stretch-mediated activation of selective MAPK subtypes and potentiation of AP-1 binding in human osteoblastic cells. Mol Med 7: 68-78.

Puklin-Faucher E, Sheetz MP (2009) The mechanical integrin cycle. J Cell Sci 122: 179-186.

Rangaswami H, Marathe N, Zhuang S, Chen Y, Yeh JC, Frangos JA, Boss GR, Pilz RB (2009) Type II cGMPdependent protein kinase mediates osteoblast mechanotransduction. J Biol Chem 284: 14796-14808.

Sharif-Naeini R, Folgering JH, Bichet D, Duprat F, Delmas P, Patel A, Honore E (2009) Sensing pressure in the cardiovascular system: Gq-coupled mechanoreceptors and TRP channels. J Mol Cell Cardiol 48: 83-89.

Silberman M, Barac YD, Yahav H, Wolfovitz E, Einav S, Resnick N, Binah O (2009) Shear stress-induced transcriptional regulation via hybrid promoters as a potential tool for promoting angiogenesis. Angiogenesis 12: 231-242.

Simonsen JL, Rosada C, Serakinci N, Justesen J, Stenderup K, Rattan SI, Jensen TG, Kassem M (2002) Telomerase expression extends the proliferative life-span and maintains the osteogenic potential of human bone marrow stromal cells. Nat Biotechnol 20: 592-596.

Sumpio BE, Chang R, Xu WJ, Wang XJ, Du W (1997) Regulation of tPA in endothelial cells exposed to cyclic strain: role of CRE, AP-2, and SSRE binding sites. Am J Physiol 273: C1441-1448. 
Wang P, Zhu F, Lee NH, Konstantopoulos K (2010) Shear-induced interleukin-6 synthesis in chondrocytes: roles of E prostanoid (EP) 2 and EP3 in $\mathrm{CAMP} /$ protein kinase A- and PI3-K/Akt-dependent NF-kappaB activation. J Biol Chem 285: 24793-24804.

Young SR, Gerard-O'Riley R, Kim JB, Pavalko FM (2009). Focal adhesion kinase is important for fluid shear stress-induced mechanotransduction in osteoblasts. J Bone Miner Res 24: 411-424.

\section{Discussion with Reviewer}

Reviewer I: How readily can the strain system be reproduced and used as a high throughput system. Is there enough cell material in each well that permits analyses (or do wells need to be pooled) - the next question is relevant to this one. What was the variability in biological measures from well to well and by spatial well position?

Authors: One well contains sufficient cells for measuring luciferase activity comparable to the cells grown in a conventional 24-well PS plate, so pooling is not necessary. Strain distribution was analyzed optically both within single trays as well as in comparison of different trays considering their spatial distribution / position on the dish. These experiments were performed with our bioreactor in conjunction with our PU-dishes thus depicting the reproducibility of stimulation parameters with the overallsystem in a setting identical to that used for biological investigations.

Reviewer I: While PU apparently permits cell attachment with substrate coating, it may be desirable or important to coat the PU with collagen, fibronectin or some other substrate as it will likely affect mechanotransduction as well as phenotypic development in the cells. Can such coating be done relatively easily?

Authors: Fibronectin coating can be done easily but was not performed in the here described experiments, since protein/peptide coating itself is known to elicit specific cellular responses which we intended to avoid. 